\title{
MRP Offsetting for Assembly Systems with Random Component Delivery Times: A Particular Case
}

\author{
Mohamed-Aly Louly ${ }^{1, *}$ and Alexandre Dolgui ${ }^{2}$ \\ ${ }^{1}$ King Saud University \\ College of Engineering - Industrial Engineering Department \\ P.O. Box 800, Riyadh 11421, Kingdom of Saudi Arabia \\ louly@ksu.edu.sa \\ ${ }^{2}$ Ecole des Mines de Saint-Étienne \\ Centre for Industrial Engineering and Computer Science \\ 158 cours Fauriel, 42023 Saint Etienne Cedex 2, France \\ dolgui@emse.fr
}

\begin{abstract}
This paper considers component supply planning for assembly systems where several types of components are needed to produce one finished product. The actual component lead times have random deviations. The aim of this study is to find the optimal MRP offsetting when the Periodic Order Quantity (POQ) policy is used. The proposed model and algorithms minimize the sum of the setup and average holding costs for the components, while satisfying a desired service level for finished product.
\end{abstract}

Keywords: Assembly Systems, Inventory Control, Stochastic Component Lead Times, Periodic Order Quantity, MRP offsetting.

\section{Introduction}

Material Requirements Planning (MRP) is a commonly accepted approach for replenishment planning in major companies. The practical aspect of MRP lies in the fact that this provides a support clear and simple to understand, as well as a powerful information system to decision making [1], [9], [10].

Nevertheless, MRP is based on the supposition that the demand and lead times are known. However, most production systems are stochastic. This is because there are some random factors and unpredictable events such as machine breakdowns, transport delays, etc. which can cause random deviations from planning [4]. Therefore, actually, the deterministic assumptions of MRP are often too restrictive. Thankfully, the MRP approach can be tailored to uncertainties by searching optimal values for its parameters [3], [11]. Thus, one of essential issues is MRP parameterization for real life companies in industrial situations. This is commonly called MRP offsetting under uncertainties.

* This work has been partially supported by Princess Fatimah Alnijris's Research Chair of Advanced Manufacturing Technology. 
Some MRP parameters are: planned lead time, safety stock, lot-sizing rule, freezing horizon, and planning horizon. There are extensive publications concerning safety stock calculation [5], [8]. In contrast, certain parameters seem not to be sufficiently examined as, for example, planned lead times. Nevertheless, this parameter (differences between due dates and release dates) is especially important for assembly systems, because for these systems all components must be present to begin the assembly, so a delay of a component blocks the entire process. The difficulty of the calculating optimal planned lead times (safety lead times) in assembly systems lies in the interdependence among different component stocks. Certainly, many types of components are needed to produce a single finished item. Therefore, the inventory level of a component depends on the stock levels of other components. Stockout for a component leads to shortage, and so decreases the service level. In addition, as the assembly process is stopped, the stocks of other components increase (because they are not used) and consequently the corresponding holding costs augment.

This problem is the subject of this paper. It was already examined in our earlier work. The case of Lot-for-Lot policy was examined in [2]. In the model proposed, the backlogs are authorized and a unit backlogging cost is supposed to be known. The objective was to minimize the sum of average backlogging and holding costs. A special case was considered in [6], when all components have identical properties, i.e. the same lead time probability distribution and unit holding cost. The optimal planned lead times were obtained using an extension of the Discrete Newsboy. This result was extended to the Periodic Order Quantity (POQ) policy in [7]. However, the assumptions used in the last two publications are relatively restrictive. Moreover, in real life applications the unit backlogging cost is difficult to ascertain. The purpose of this paper is to extend these models to a more general case of POQ policy with service level constraints and different holding and setup costs.

\section{Objective of this Study}

Each MRP table has several parameters: lot-sizing rule; planned lead time for the time phasing, safety stock, etc.

In this paper, the POQ lot-sizing rule is considered and only the following essential parameters are optimized (due to of their importance):

periodicity $(p)$;

planned lead times $\left(x_{i}\right)$.

It is clear that, for assembly systems, the parameters $x_{i}$ and the periodicity $p$ cannot be calculated separately for each component type (if we search for optimal solutions). Indeed, in assembly systems, the difficulty of determining optimal component planned lead times resides in the interdependence among different component inventories. Many types of components are needed to produce one product; therefore, the inventories of the different components become dependent.

In this paper, we consider assembly systems with one-level BOM when the POQ lot-sizing policy is used. Components are ordered every $p$ periods. The periodicity is the same for all component types. The goal is to search for the optimal values of the parameters $p$ and $x_{i}, i=1,2, \ldots, n$ minimizing the sum of the setup and average holding 
costs for the components, while satisfying a desired service level for finished product as well as taking into account the interdependence among the inventories of the different components.

\section{Cost and Service Level Calculation}

The POQ lot-sizing rule is used with periodicity $p$ common for all component types ( $p$ is a decision variable). The orders for components are made at the beginning of the periods $k p+1, k=0,1,2, \ldots$, and there is no order made in the periods $k p+r, r=2,3, \ldots, p$.

The following additional notations are used:

$h_{i} \quad$ unit holding cost for component $i$

$c \quad$ setup cost, i.e. the cost incurred each time a replenishment order is made

$L_{i} \quad$ probability distribution for the component $i$ lead time

$u_{i} \quad$ upper value of lead time distribution for component $i$

$L_{i}^{k} \quad$ lead time of the components $i$ ordered at the beginning of the period $k$

$D$ demand for finished product per period

$a_{i} \quad$ quantity of component $i$ needed to assemble the finished product

$p \quad$ supply periodicity

$Q_{i} \quad$ supply order quantity for component $i$

$x_{i} \quad$ planned lead time for component $i$

$1-\varepsilon$ objective service level

The demand is constant, therefore, the supply orders $Q_{\mathrm{i}}$ of components $i$ are also constant $Q_{i}=a_{i} D p$. The finished products are delivered at the end of each period and unsatisfied part of demand is backordered and has to be satisfied during the subsequent periods.

In the considered model, the quantities ordered are the same, so the planned lead times give also initial inventories. Thus, the aim of this study can be expressed in other terms: to find the optimal values of the initial inventories $a_{i} D x_{i}$ and parameter $p$, where $x_{i}, i=1,2, \ldots, n$, are the planned lead times.

This approach takes into account the major factors of the supply planning in assembly systems with random lead times to obtain an efficient optimization algorithm for planned lead times and the periodicity calculation.

As aforementioned, a particular case of this model was earlier considered in [7], where all components have identical properties, i.e., the same lead time probability distribution and the same unit holding cost. The new techniques proposed in this paper were developed without these restrictive assumptions.

For the considered model, given that the maximal value of the component $i$ lead time is equal to $u_{i}$, only the orders made in the previous $u_{i}-1$ periods may not have arrived yet. The orders made before have already arrived. Therefore, the number $N_{i}^{p, m}$ of orders for the component $i$ which are in waiting at the end of the period $m=k p+r$ is easy to calculate.

Let

$$
L_{i}^{m+1-j}, j=r, r+p, r+2 p, \ldots, r+\frac{u_{i}-1-r}{p} p,
$$


be the lead times of the orders made at the beginning of the periods $k p+1,(k$ 1) $p+1, \ldots,\left(k-\frac{u_{i}-1-r}{p}\right) p+1$.

If $L_{i}^{m+1-j}>j$, then the order made in the period $m+1-j$ is delivered after the end of the period $m$.

Let $1_{E}$ be the binary function equal to 1 when the expression $E$ is true and equal to 0 otherwise. Therefore, if $1_{L_{i}^{m+1-j}>j}$ is equal to 1 , then the order made at the period $m+1-j$ is delivered after the end of the period $m$. Thus, the random variables $N_{i}^{p, m}$ can be represented as follows:

$$
\begin{gathered}
N_{i}^{p, k p+r}=\sum_{j=0}^{\frac{u_{i}-1-r}{p}} L_{i}^{(k-j) p+1}>j p+r \quad i=1, \cdots, n \\
k \geq 0, p \in\left\{1,2, . ., u_{i}-1\right\}, r \in\{1,2, . ., p\}
\end{gathered}
$$

The variables $N_{i}^{p, m}$ are independent for different types of components, and also independent from the decision variables $x_{i}$. Thus, they can be used to derive closed forms for the shortage level and cost [7].

The average cost has the following closed form:

$$
\begin{gathered}
C(X, p)=\frac{1}{p} \sum_{r=1}^{p} E\left(C\left(X, p, N^{p, k p+r}\right)\right)=\frac{c}{p}+ \\
\frac{p-1}{2} H+\sum_{i=1}^{n} h_{i}\left(x_{i}-E\left(N_{i}^{p}\right)\right)+H \sum_{k \geq 0}\left(1-\frac{1}{p} \sum_{r=1}^{p} \prod_{i=1}^{n} F_{i}^{p, r}\left(\frac{x_{i}+k-r+p}{p}\right)\right)
\end{gathered}
$$

The average number of shortages has the following closed form:

$$
\begin{gathered}
S(X, p)=\frac{1}{p} \sum_{r=1}^{p} \operatorname{Pr}\left[\max _{i=1, \cdots, n}\left(p N_{i}^{p, k p+r}+r-p-x_{i}\right)^{+}>0\right]= \\
1-\frac{1}{p} \sum_{r=1}^{p} \prod_{i=1}^{n} F_{i}^{p, r}\left(\frac{x_{i}-r+p}{p}\right)
\end{gathered}
$$

\section{Cost Optimization under Service Level Constraint}

The optimization problem can then be written as follows:

$$
\text { Minimize } C(X, p)
$$

Subject to:

$$
\frac{1}{p} \sum_{r=1}^{p} \prod_{i=1}^{n} F_{i}^{p, r}\left(\frac{x_{i}-r+p}{p}\right) \geq 1-\varepsilon
$$




$$
\begin{gathered}
N_{i}^{p}=\sum_{r=1}^{p} N_{i}^{p, r} \\
F_{i}^{p, r}(x)=\operatorname{Pr}\left(N_{i}^{p, r} \leq x\right) \\
H=\sum_{i=1}^{n} h_{i} \\
0 \leq x_{i} \leq u_{i}-1, \quad i=1, \ldots, n \\
1 \leq p \leq u-1
\end{gathered}
$$

The optimization problem (4)-(10) seems difficult to solve because of the nonlinearity of the objective function and the fact that the decision variables are integers. Nevertheless, in an earlier work [12] we already solved a similar problem: minimizing the sum of holding, setup and backlogging (instead of the service level constraint) costs. The approach was based on the partial incremental functions defined as follows:

$$
\begin{aligned}
& G_{i}^{+}(X, p)=C\left(x_{1}, \ldots, x_{i}+1, \ldots, x_{n}, p\right)-C\left(x_{1}, \ldots, x_{i}, \ldots, x_{n}, p\right), \\
& G_{i}^{-}(X, p)=C\left(x_{1}, \ldots, x_{i}-1, \ldots, x_{n}, p\right)-C\left(x_{1}, \ldots, x_{i}, \ldots, x_{n}, p\right) .
\end{aligned}
$$

It was proved that $G_{i}^{+}(X, p)$ is increasing on $x_{i}$ and decreasing on $x_{j}$ for $j$ different from $i$. Inversely, $G_{i}^{-}(X, p)$ is decreasing on $x_{i}$ and increasing on $x_{j}$ for $j$ different from $i$.

These properties can be easily extended for the objective function considered in this paper (the sum of holding and setup costs) with service level constraint and used to prove the following dominance properties:

If $G_{i}^{+}(A, p)<0$, then each solution $(X, p)$ with $x_{i}=a_{i}$ is dominated,

If $G_{i}^{-}(B, b)<0$ and the vector $\left(a_{1}, \ldots, a_{i-1}, b_{i}-1, a_{i+1}, \ldots, x_{n}\right)$ satisfies the desired service level, see constraint (9), then each solution $(X, p)$ with $x_{i}=b_{i}$ is dominated.

In addition, the following lower bound on the objective function in the space $[A, B] \times\{p\}$ can be proven:

$$
L B(p)=C(A, p)+\sum_{i=1}^{n}\left(b_{i}-a_{i}\right) \min \left(G_{i}^{+}\left(b_{1}, \ldots, b_{i-1}, a_{i}, \ldots, a_{n}, p\right), 0\right)
$$

Let us present the space of all possible values of $x_{i}$ by $[A, B]$, where $A=\left(a_{1}, a_{2}, \ldots\right.$, $\left.a_{n}\right), B=\left(b_{1}, b_{2}, \ldots, b_{n}\right)$, and $a_{i}, b_{i}$ are minimal and maximal possible values for $x_{i}$, respectively, i.e. $a_{i} \leq x_{i} \leq b_{i}$.

A Branch and Bound (B\&B) approach can be used. Each node of the Branch and Bound tree represents a solution space $[A, B] \times p$. Two cut procedures are developed based on the aforementioned dominance properties. They are applied to each current 
solution space $[A, B] \times p$ (to each current node). The forward cut procedure replaces $A$ by a larger vector, while the backward cut procedure replaces $B$ by a smaller vector. A procedure is also developed to calculate lower bounds for any solution space $[A, B] \times p$, i.e., any branch tree node, using $L B(p)$ see (11). To calculate an Upper Bound for a given node $[A, B] \times p$, a heuristic method consisting in a variant of depth first search can be used. It partitions the solution space (current node) into two subspaces (nodes) and chooses as current node the node that has the best feasible solution at one of its two extremities, and so on. A root upper bound is also calculated by exploring all the promising values of the parameter $p$.

\section{Algorithm B\&B:}

For each periodicity $p$ do:

- Activate the solution space corresponding to the periodicity $p$. This initial space is represented by one node corresponding to all possible lead time values.

- Reduce the size of this node using dominance properties.

While the solution space is not empty do:

- Activate the node corresponding to the subset having the largest number of solutions.

- $\quad$ Divide this node into two new nodes (subsets).

- $\quad$ Reduce the size of the new nodes using dominance properties.

- If the reduced subsets contain feasible and not dominated solutions, then: add them to the solution space.

- If the processing of the new nodes gives a better solution than the current best one, then: update the current best solution and delete the nodes having their lower bound larger than the current best solution.

\section{End while}

\section{End for}

The algorithm is applied for each value of $p$ whose initial solution space $[A, B] \times p$ is not dominated. This B\&B algorithm is a variant of width first search that consists in choosing for extension the node that contains the maximum number of solutions.

We studied an example with $n=10$ different types of components. The maximum value of the lead time was equal to $10(u=10)$. The unit holding costs $h_{i}$ were chosen as follows: 9, 2, 4, 6, 4, 3, 10, 5, 6, and 2. Setup (ordering) cost $c$ was equal to 100 . The required service level was 0.99 .

For this example, the optimal solution was obtained after 301 iterations of the B\&B algorithm, where, the cost is equal to 266.652 , periodicity $p$ is 2 , and values of planned lead times for the considered ten types on components are $8,9,9,8,8,8,8$, $7,7,8$, respectively.

\section{Conclusions}

This paper further develops the models of our previous publications while considering the case of POQ policy. The objective is to determine the values of the following two types of MRP parameters for all components: order periodicity and planned lead times, minimizing the sum of average holding cost for the components and setup 
costs. There are no restrictive assumptions on the probability distributions and unit holding costs. In addition the backlogging cost is replaced with a service level constraint, which is a more realistic parameter.

The model presented in this paper uses less restrictive assumptions than previous models known in literature. This is a multi-period model with no restriction on the number of components and where lead time density function for each component may differ from the density functions of other components. All possible distributions can be used for component lead times. The decision variables are integer; they represent the periodicity and planned lead times for components. Branch and Bound approach is developed. The experimental study shows that the proposed B\&B algorithm is very fast. It will always find the optimal solution within a very short computing time.

The proposed model and algorithms can be used in many industrial situations. For example, often security coefficients are introduced to calculate the planned lead time for unreliable suppliers in an MRP environment. In this case, planned lead times are equal to contractual (or forecasted) lead times multiplied by the security coefficients. These coefficients are empiric but anticipate the delays by creating safety lead times. The more unreliable a supplier is, the larger its coefficient. The model and algorithms suggested in this paper can be used to better estimate these coefficients basing on statistics on the procurement lead times for each supplier and taking into account the holding and setup costs, as well as a service level constraint.

Nevertheless, the model proposed keeps some restrictive assumptions as: fixed and constant demand for the finished product (for a given period), one-level bill of material, same order cost and periodicity for all components, etc. Therefore, for some actual industrial cases, the solution obtained can be approximate and not optimal. Future research will be focused on the study of how to relax some of these assumptions, for example, examining multi-level bills of material (BOM).

Concerning the assumption of constant demand, note that this model should be used with different possible values of the demand to examine the sensitivity of the obtained parameters to said values. If the parameter values are significantly different for the given demand levels, the approach by scenarios can be applied to choose the parameter values. In addition, the demand variations can be decoupled from planned lead time calculation by using safety stocks. This is another perspective for future research.

\section{References}

1. Axsäter, S.: Inventory Control, 2nd edn. Springer, Heidelberg (2006)

2. Dolgui, A., Louly, M.A.: A Model for Supply Planning under Lead Time Uncertainty. Int. J. Prod. Econ. 78, 145-152 (2002)

3. Dolgui, A., Prodhon, C.: Supply Planning under Uncertainties in MRP Environments: A State of the Art. Ann. Rev. Contr. 31(2), 269-279 (2007)

4. Koh, S.C.L., Saad, S.M.: MRP-controlled Manufacturing Environment Disturbed by Uncertainty. Robot. Comput. Integr. Manuf. 19, 157-171 (2003)

5. Lee, H.L., Nahmias, S.: Single-product, Single-Location Models. In: Graves, S.C., Rinnooy Kan, A.H.G., Zipkin, P.H. (eds.) Handbooks in Operations Research and Management Science, vol. 4, pp. 3-57. North-Holland, Amsterdam (1993) 
6. Louly, M.A., Dolgui, A.: Newsboy Model for Supply Planning of Assembly Systems. Int. J. Prod. Res. 40, 4401-4414 (2002)

7. Louly, M.A., Dolgui, A.: The MPS Parameterization under Lead Time Uncertainty. Int. J. Prod. Econ. 90, 369-376 (2004)

8. Porteus, E.L.: Stochastic Inventory Theory. In: Heyman, D.P., Sobel, M.J. (eds.) Handbooks in Operations Research and Management Science, vol. 2, pp. 605-652. Elsevier Science Publishers, Amsterdam (1990)

9. Sipper, D., Bulfin Jr., R.L.: Production: Planning, Control and Integration. McGraw Hill, New York (1998)

10. Tempelmeier, H.: Inventory Management in Supply Networks: Problems, Models, Solutions, Books on Demand GmbH (2006)

11. Whybark, D.C., Wiliams, J.G.: Material Requirement under Uncertainty. Dec. Sci. 7, 595606 (1976)

12. Louly, M.A., Dolgui, A.: An Optimization Approach for Time Phasing in MRP Systems. In: Preprints of the Fifteen International Working Seminar on Production Economics, Innsbruck, Austria, March 3-7, vol. 3, pp. 273-280 (2008) 